DOI: 10.12731/2658-6649-2019-11-3-103-120

УДК 616.24-007-06; 616.12-008.331.1-06

\title{
ВЛИЯНИЕ СОЦИАЛЬНО-ЭКОНОМИЧЕСКИХ ФАКТОРОВ НА КАЧЕСТВО ЖИЗНИ БОЛЬНЫХ ХРОНИЧЕСКОЙ ОБСТРУКТИВНОЙ БОЛЕЗНЬЮ ЛЕГКИХ В УСЛОВИЯХ КОМОРБИДНОСТИ
}

\author{
Козлов Е.В., Деревянных Е.В., Балашова Н.А., \\ Яскевич Р.А., Москаленко О.Л.
}

Цель. Изучение влияния сочииально-экономических факторов на качество жизни у больных хронической обструктивной болезнью легких (ХОБЛ) в условиях коморбидности с артериальной гипертонией (АГ).

Материалы и методы. В исследование было включено 136 пациентов мужского пола, средний возраст 61 лет. Из них паџиентов с ХОБЛ в сочетании с АГ-- 63 человека, пациентов с ХОБЛ без АГ 29 человек. Группу сравнения составили 44 пациента с АГ без ХОБЛ. Исследование качества жизни проводилось методом самоанкетирования с использованием общего вопросника MOS SF-36, так же учитывали сочиально-экономический статус пацичентов.

Результаты. В ходе проведенного исследования выявлено снижение КЖ по всем параметрам у пациентов с ХОБЛ и в большей степени у лии при коморбидности ХОБЛ и АГ, что вероятнее всего связано с прогрессированием и большей выраженностью клинической симптоматики. Наиболее значимой сферой отношений на всех стадиях является сочиально-экономический статус, значимость которого увеличивается по мере нарастания тяжести болезни. Более низкие значения по шкалам, характеризующим как физический, так и психосоциальный статус респондентов, регистрировались у неработающих лиц. КЖ прямо пропориионально снижалось ( $<<0,05)$, при наличии группы инвалидности, при чем более выраженно у пачиентов при коморбидности ХОБЛ и АГ. Рассматривая влияние среднего прожиточного минимума, из расчета на одного члена семьи, на уровень КЖ, статистически значимые внутригрупповые отличия были получены среди пациентов с изолированной ХОБЛ по шкалам общего здоровья и физического функиионирования $(p<0,05)$. 
Заключение. Сочиально-экономический статус оказывает существенное влияние на КЖ респондентов, при чем как в целом, так и на отдельно составляюшие его части.

Ключевые слова: хроническая обструктивная болезнь легких; артериальная гипертония; качество жизни.

\title{
THE EFFECT OF SOCIO-ECONOMIC FACTORS ON THE QUALITY OF LIFE IN PATIENTS WITH CHRONIC OBSTRUCTIVE LUNG DISEASE IN CONDITIONS OF COMORBIDITY
}

\author{
Kozlov E.V., Derevyanykh E.V., Balashova N.A., \\ Yaskevich R.A., Moskalenko O.L.
}

The purpose of the study. The study of the influence of socio-economic factors on the quality of life in men with chronic obstructive pulmonary disease (COPD) in conditions of comorbidity with arterial hypertension $(A H)$.

Materials and methods. The study included 136 male patients, mean age 61 years. Of these patients with COPD in combination with hypertension - 63 people, patients with COPD without hypertension 29 people. The comparison group consisted of 44 patients with hypertension without COPD. The study of the quality of life was carried out using the self-questioning method using the common MOS SF-36 questionnaire, as well as taking into account the socioeconomic status of patients.

Results. In the course of the study, there was a decrease in $Q O L$ in all parameters in patients with COPD and to a greater extent in individuals with comorbidity of COPD and $A H$, which is most likely associated with progression and greater severity of clinical symptoms. The most significant area of relations at all stages is the socioeconomic status, the importance of which increases as the severity of the disease increases. Lower values on the scales characterizing both the physical and psychosocial status of the respondents were recorded with non-working persons. QOL was directly proportional $(p<0.05)$ in the presence of a disability group, and it was more pronounced in patients with comorbidity of COPD and AH. Considering the effect of the average subsistence minimum, based on one family member, on the QOL level, statistically significant intragroup differences were obtained among patients with isolated COPD on the scales of general health and physical functioning $(p<0.05)$. 
Conclusion. Socio-economic status has a significant impact on the respondents' $Q O L$, both in general and in the individual components of it.

Keywords: chronic obstructive pulmonary disease; arterial hypertension; comorbidity; the quality of life.

\section{Введение}

Хроническая обструктивная болезнь легких (ХОБЛ) является одним из наиболее распространенных респираторных заболеваний $[4$, с. $57 ; 11$, c. 180-199]. Согласно прогнозам Всемирной организации здравоохранения (ВО3), она станет третьей по значимости причиной смерти в мире после сердечно-сосудистых и онкологических заболеваний в 2030 году $[4$, c. 57]. Сердечно-сосудистая патология является самой серьезной болезнью, сосуществующей с ХОБЛ [9, с. 56-66; 10, с. 19-25; 14, с. 66-69]. Большое количество проведенных исследований подтверждает актуальность изучения качества жизни (КЖ) актуально в различных областях здравоохранения [1, с. 204-222; 7, с. 65-71; 17, с. 73-74; 18, с. 81-83] в том числе и в пульмонологии, особенно у больных с хроническими нарушениями бронхиальной проходимости [12, с. 6-13; 23, с. 125-129]. Исследования КЖ, проводимые среди пациентов с ХОБЛ, приобретают все большее значение в качестве ценного дополнения к оценке клинического состояния пациента, эффективности терапии, образования и профилактики, а также клинической оценки препаратов [2, с. $54-55 ; 3$, с. 31-35; 8, с. 9-14; 23, с. 125-129]. ВОЗ определяет КЖ как восприятие людьми своего положения в жизни в контексте культуры и систем ценностей, в которых они живут, и в отношении их целей, ожиданий, стандартов и проблем $[13$, с. $64 ; 15$, с. 2$]$. Уровень КЖ и состояние здоровья в хронических условиях измеряются как с помощью общих, так и с помощью специальных вопросников [15, с. 2; 24, с. 667-675]. ХОБЛ как хроническое заболевание оказывает влияние на социальную, психологическую и экономическую сферы жизни [7, с. 65-71; 20, эл. ресурс; 22, с. 2189-2196]. Социальноэкономический статус означает положение индивида в обществе, которое в основном обусловлено образованием, доходом, профессиональной деятельностью и фактической профессией [26, с. 19-30]. Имеются доказательства того, что различия в состоянии здоровья могут зависеть от доходов, благосостояния, образования, профессии и социально-экономических особенностей окружающей среды [5, с. 22-23; 6, с. 24-25; 19, с. 279]. Поэтому представляется целесообразным анализировать влияние отдельных социально-экономических факторов, а не общего социально-экономиче- 
ского статуса [26, с. 19-30]. Кроме того, ряд исследователей указывают на необходимость рассмотрения социально-экономического статуса как переменной в измерении КЖ [26, с. 19-30]. По мнению этих авторов, низкий социально-экономический статус связан с более высокими показателями заболеваемости и смертности вследствие хронических заболеваний, включая сердечно-сосудистые заболевания, ХОБЛ, астму и диабет [20, эл. pecypc; 25, с. 283-287]. Знания о взаимосвязи между ХОБЛ и конкретными социально-экономическими элементами по-прежнему недостаточны, что обусловливает необходимость проведения исследований в этой области.

\section{Цель исследования}

Оценить влияние социально-экономических факторов на КЖ у больных с ХОБЛ при коморбидности с АГ.

\section{Материалы и методы}

В исследование было включено 136 пациентов мужского пола, средний возраст 61 [57-68] лет. Из них пациенты с ХОБЛ в сочетании с АГ - 63 чел. (средний возраст 64 [58-70] лет), пациентов с ХОБЛ без АГ 29 чел. (средний возраст 59 [52-69] лет). Группу сравнения составили 44 пациента с АГ без ХОБЛ (средний возраст 60 [58-64] лет). Протокол исследования включал: клинические, инструментальные и лабораторные методы исследования. Верификация диагноза ХОБЛ и оценка степени тяжести проводилась в соответствии с критериями GOLD (Global Initiative for Chronic Obstructive Lung Disease, update 2014) [4, c. 57]. Верификацию АГ проводили согласно Рекомендациям по диагностике и лечению АГ Всероссийского научного общества кардиологов (ВНОК,2013) [16, с. 5-34].

Исследование КЖ проводилось методом самоанкетирования с использованием общего «Краткого вопросника оценки статуса здоровья» (официального русскоязычного аналога вопросника MOS SF-36 [21, с. 77-84]. На основании результатов анкетирования по вопроснику MOS SF-36 проводился расчет 5 показателей, характеризующих физический статус респондента (физическая активность - ФА, роль физических проблем в ограничении жизнедеятельности - РФ, боль - Б, общее здоровье - ОЗ, жизнеспособность - ЖС), и 5 параметров, отражающих его психосоциальный статус (роль эмоциональных проблем в ограничении жизнедеятельности - РЭ, социальная активность - СА, психическое здоровье - ПЗ, общее здоровье - ОЗ и жизнеспособность - ЖС). Два последних показателя определяются как физическим, так и психическим статусом человека. 
Опросник SF-36 позволяет оценивать КЖ по 100-балльной шкале. Такие шкалы как ФА, О3, ЖС, ПЗ, СА, оцениваются как «прямые»: чем выше значение, тем лучше КЖ пациента. Шкалы РФ, Б, РЭ оцениваются как «обратные» критерии: чем выше значение, тем менее выражено его влияние на КЖ человека. Обработку полученных данных проводили при помощи пакета прикладных программ Statistica 6.0 (Statsoft Inc., США). Количественные значения представлялись в виде медианы (Me) и межквартильного интервала (Q25-Q75). Достоверность различий между показателями независимых выборок оценивали по непараметрическому критерию Манна-Уитни. При сравнении групп по качественному признаку использовали критерий 2. Различия считали статистически значимыми при $р<0,05$.

\section{Результаты и обсуждение}

При исследовании влияния социально-экономических факторов на показатели КЖ у обследуемых лиц установлено, что более низкие значения по всем шкалам опросника SF-36 отмечались у неработающих мужчин с ХОБЛ как с АГ, так и без неё (табл. 1).

Таблица №1.

Сравнительный анализ показателей качества жизни

в зависимости от социального статуса $\left(\mathrm{Me}\left[\mathrm{Q}_{25}-\mathrm{Q}_{75}\right]\right)$

\begin{tabular}{|c|c|c|c|c|c|c|}
\hline \multirow{2}{*}{ КЖ } & \multicolumn{2}{|c|}{$\begin{array}{c}1-я \text { группа ХОБЛ } \\
(\mathrm{n}=28)\end{array}$} & \multicolumn{2}{|c|}{$\begin{array}{c}2 \text { 2-я группа ХОБЛ + АГ } \\
(\mathrm{n}=57)\end{array}$} & \multicolumn{2}{|c|}{$\begin{array}{c}\text { 3-я группа } А Г \\
(\mathrm{n}=40)\end{array}$} \\
\hline & $\begin{array}{l}\text { I. Работаю- } \\
\text { щие }\end{array}$ & $\begin{array}{c}\begin{array}{c}\text { II. Неработа- } \\
\text { ющие }\end{array} \\
\end{array}$ & $\begin{array}{l}\text { I. Работаю- } \\
\text { щие }\end{array}$ & $\begin{array}{l}\text { II. Неработа- } \\
\text { ющие }\end{array}$ & $\begin{array}{l}\text { I. Работаю- } \\
\text { щие }\end{array}$ & $\begin{array}{l}\text { II. Неработа- } \\
\text { ющие }\end{array}$ \\
\hline \multirow[t]{2}{*}{ O3 } & $\begin{array}{c}66,0 \\
{[50,0-77,0]}\end{array}$ & $\begin{array}{c}35,0 \\
{[25,0-50,0]}\end{array}$ & $\begin{array}{c}30,0 \\
{[20,0-35,0]}\end{array}$ & $\begin{array}{c}35,0 \\
{[20,0-45,0]}\end{array}$ & $\begin{array}{c}60,0 \\
{[52,0-75,0]}\end{array}$ & $\begin{array}{c}61,0 \\
{[50,0-65,0]}\end{array}$ \\
\hline & \multicolumn{2}{|c|}{$\mathrm{p}=0,02$} & \multicolumn{2}{|c|}{$\mathrm{p}=0,4$} & \multicolumn{2}{|c|}{$\mathrm{p}=0,7$} \\
\hline \multicolumn{7}{|c|}{ pI 1,2=0,009 pI $1,3=0,8$ pI $\mathbf{2 , 3}=\mathbf{0 , 0 0 1}$ pII $1,2=0,6$ pII $\mathbf{1 , 3}=\mathbf{0 , 0 0 8}$ pII $\mathbf{2 , 3}=\mathbf{0 , 0 0 2}$} \\
\hline \multirow[t]{2}{*}{$\Phi A$} & $\begin{array}{c}76,5 \\
{[35,0-95,0]}\end{array}$ & $\begin{array}{c}32,5 \\
{[25,0-60,0]} \\
\end{array}$ & $\begin{array}{c}42,5 \\
{[32,5-65,0]} \\
\end{array}$ & $\begin{array}{c}35,0 \\
{[20,0-40,0]} \\
\end{array}$ & $\begin{array}{c}92,5 \\
{[80,0-95,0]}\end{array}$ & $\begin{array}{c}92,5 \\
{[85,0-95,0]}\end{array}$ \\
\hline & \multicolumn{2}{|c|}{$p=0,04$} & \multicolumn{2}{|c|}{$\mathrm{p}=0,06$} & \multicolumn{2}{|c|}{$\mathrm{p}=0,8$} \\
\hline \multicolumn{7}{|c|}{ pI $1,2=0,09$ pI 1,3=0,05 pI 2,3=0,001 pII $1,2=0,3$ pII $\mathbf{1 , 3}=\mathbf{0 , 0 1}$ pII 2,3=0,002 } \\
\hline \multirow[t]{2}{*}{ РФ } & $\begin{array}{c}62,5 \\
{[10,0-90,0]}\end{array}$ & $\begin{array}{c}12,5 \\
{[10,0-50,0]}\end{array}$ & $\begin{array}{c}15,0 \\
{[10,0-50,0]}\end{array}$ & $\begin{array}{c}10,0 \\
{[5,0-25,0]}\end{array}$ & $\begin{array}{c}75,0 \\
{[25,0-90,0]}\end{array}$ & $\begin{array}{c}90,0 \\
{[12,5-95,0]}\end{array}$ \\
\hline & \multicolumn{2}{|c|}{$\mathrm{p}=\mathbf{0 , 0 5}$} & \multicolumn{2}{|c|}{$\mathrm{p}=0,6$} & \multicolumn{2}{|c|}{$\mathrm{p}=0,5$} \\
\hline \multicolumn{7}{|c|}{ pI $1,2=0,07$ pI $1,3=0,9$ pI $\mathbf{2 , 3}=\mathbf{0 , 0 1}$ pII $1,2=0,4$ pII $\mathbf{1 , 3}=\mathbf{0 , 0 5}$ pII $\mathbf{2 , 3}=\mathbf{0 , 0 2}$} \\
\hline \multirow[t]{2}{*}{ РЭ } & $\begin{array}{c}90,0 \\
{[34,0-95,0]}\end{array}$ & $\begin{array}{c}34,0 \\
{[10,0-67,0]}\end{array}$ & $\begin{array}{c}67,0 \\
{[34,0-90,0]} \\
\end{array}$ & $\begin{array}{c}34,0 \\
{[15,0-40,0]}\end{array}$ & $\begin{array}{c}80,0 \\
{[34,0-90,0]}\end{array}$ & $\begin{array}{c}90,0 \\
{[67,0-95,0]} \\
\end{array}$ \\
\hline & \multicolumn{2}{|c|}{$\mathrm{p}=\mathbf{0 , 0 1}$} & \multicolumn{2}{|c|}{$\mathrm{p}=\mathbf{0 , 0 3}$} & \multicolumn{2}{|c|}{$\mathrm{p}=0,6$} \\
\hline
\end{tabular}




\begin{tabular}{|c|c|c|c|c|c|c|}
\hline & & & & & \multicolumn{2}{|c|}{ Окончание табл. 1} \\
\hline \multirow[t]{2}{*}{$\mathrm{CA}$} & $\begin{array}{c}50,0 \\
{[38,0-60,0]}\end{array}$ & $\begin{array}{c}50,0 \\
38,0-55,0]\end{array}$ & $\begin{array}{c}44,0 \\
{[38,0-56,5]}\end{array}$ & $\begin{array}{c}50,0 \\
{[38,0-55,0]}\end{array}$ & $\begin{array}{c}50,0 \\
{[38,0-60,0]}\end{array}$ & $\begin{array}{c}44,0 \\
{[38,0-50,0]}\end{array}$ \\
\hline & \multicolumn{2}{|c|}{$\mathrm{p}=0,8$} & \multicolumn{2}{|c|}{$\mathrm{p}=0,5$} & \multicolumn{2}{|c|}{$\mathrm{p}=0,2$} \\
\hline \multicolumn{7}{|c|}{ pI $1,2=0,7$ pI $1,3=0,3$ pI $2,3=0,6$ pII $1,2=0,7$ pII $1,3=0,5$ pII $2,3=0,7$} \\
\hline \multirow[t]{2}{*}{ Б } & $\begin{array}{c}58,0 \\
{[31,0-84,0]}\end{array}$ & $\begin{array}{c}46,0 \\
{[31,0-74,0]}\end{array}$ & $\begin{array}{c}46,5 \\
{[41,0-62,0]}\end{array}$ & $\begin{array}{c}41,0 \\
{[31,0-62,0]}\end{array}$ & $\begin{array}{c}67,0 \\
{[41,0-90,0]}\end{array}$ & $\begin{array}{c}68,0 \\
{[32,0-84,0]}\end{array}$ \\
\hline & \multicolumn{2}{|c|}{$\mathrm{p}=0,5$} & \multicolumn{2}{|c|}{$\mathrm{p}=0,5$} & \multicolumn{2}{|c|}{$\mathrm{p}=0,6$} \\
\hline \multicolumn{7}{|c|}{ pI $1,2=0,5$ pI $1,3=0,4$ pI $2,3=0,06$ pII $1,2=0,5$ pII $1,3=0,4$ pII $2,3=0,2$} \\
\hline \multirow[t]{2}{*}{ ЖС } & $\begin{array}{c}70,0 \\
{[15,0-75,0]}\end{array}$ & $\begin{array}{c}37,5 \\
{[25,0-55,0]}\end{array}$ & $\begin{array}{c}42,5 \\
{[30,0-55,0]}\end{array}$ & $\begin{array}{c}35,0 \\
{[25,0-50,0]}\end{array}$ & $\begin{array}{c}62,5 \\
{[50,0-75,0]}\end{array}$ & $\begin{array}{c}75,0 \\
{[70,0-85,0]} \\
\end{array}$ \\
\hline & \multicolumn{2}{|c|}{$\mathrm{p}=0,5$} & \multicolumn{2}{|c|}{$\mathrm{p}=0,6$} & \multicolumn{2}{|c|}{$\mathrm{p}=0,2$} \\
\hline \multicolumn{7}{|c|}{ pI 1,2=0,4 pI 1,3=0,7 pI 2,3=0,004 pII 1,2=0,7 pII 1,3=0,01 pII 2,3=0,006 } \\
\hline \multirow[t]{2}{*}{ ПЗ } & $\begin{array}{c}75,5 \\
{[28,0-84,0]}\end{array}$ & $\begin{array}{c}44,0 \\
{[40,0-68,0]}\end{array}$ & $\begin{array}{c}46,0 \\
{[36,0-70,0]}\end{array}$ & $\begin{array}{c}48,0 \\
{[28,0-60,0]}\end{array}$ & $\begin{array}{c}64,0 \\
{[48,0-80,0]} \\
\end{array}$ & $\begin{array}{c}64,0 \\
{[44,0-80,0]} \\
\end{array}$ \\
\hline & \multicolumn{2}{|c|}{$\mathrm{p}=0,2$} & \multicolumn{2}{|c|}{$\mathrm{p}=0,5$} & \multicolumn{2}{|c|}{$\mathrm{p}=0,9$} \\
\hline & pI $1,2=0$ & $3 \mathrm{pI} 1,3=0,8$ & $2,3=0,06 \mathrm{pII}$ & $2=0,6$ pII 1,3 & 0,09 pII $2,3=$ & \\
\hline
\end{tabular}

Примечание: О3 - общее здоровье, ФА - физическая активность, РФ - роль физических проблем в ограничении жизнедеятельности, РЭ-роль эмоциональных проблем в ограничении жизнедеятельности, СА - социальная активность, Б - боль, ЖС - жизнеспособность, ПЗ - психическое здоровье.

Установлено что у этих пациентов по отдельным шкалам, характеризующим физический и психосоциальный статус, отмечались внутригрупповые статистически значимые отличия. У пациентов $1^{- \text {й }} г$ гуппы чаще выявлялись статистически значимые отличия $(\mathrm{p}<0,05)$ среди работающих и неработающих лиц по таким шкалам, как ОЗ, ФА, РФ, РЭ, что может быть обусловлено тяжестью течения заболевания. Так, у работающих пациентов с ХОБЛ выявлены менее выраженные функциональные нарушения легких по данным спирографии: - $53,0[36,0-70,0]$ и $31,5[28,0-44,0](\mathrm{p}=0,007)$ соответственно. У пациентов 2 -й группы внутригрупповые показатели КЖ были значительно снижены, как у работающих, так и неработающих лиц. Исключением были значения по шкале РЭ, которые составили у работающих 67,0 [34,0-90,0] и неработающих 34,0 [15,0-40,0] (p=0,03) балла. По этой шкале оценивается влияние эмоционального состояния, обследуемого на качество, затрату времени и объем проделанной работы. Статистически значимых отличий по функциональному нарушению легких зарегистрировано не было - 32,0 $[26,5-50,0]$ и $30,0[23,5-41,0](p=0,4)$, но отмечалась тенденция к более низким значениям у неработающих пациентов. При анализе межгрупповых сравнений $1^{-и ̆}$ и $2^{-и ̆}$ различий показателей КЖ выявлено не было $(\mathrm{p}>0,05)$. При сравнении $1^{\text {-й }}$ 
и $2^{\text {-й }}$ групп пациентов с АГ без ХОБЛотмечалась тенденция к более низким показателям КЖ по всем шкалам опросника SF-36. У пациентов, страдающих ХОБЛ, статистически значимые различия выявлены по всем шкалам ( $<<0,05)$ среди неработающих лиц, за исключением шкал СА, Б и ПЗ, по которым отмечается тенденция к более низким показателям у мужчин, страдающих ХОБЛ с АГ и без АГ. В связи с чем можно предположить, что ХОБЛ в значительной мере оказывает влияние на снижение КЖ пациентов, при этом более низкие значения наблюдаются у неработающих лиц при коморбидности изучаемых заболеваний. Проведен анализ показателей КЖ среди пациентов ХОБЛ с АГ и без АГ в зависимости от наличия группы инвалидности пациентов (табл. 2). Лица с изолированной АГ не анализировались, так как из всей группы с инвалидностью был только 1 человек. При сравнении пациентов без группы инвалидности с лицами, имеющими 2 и 3 группу, выявлено прямо пропорциональное снижение показателей КЖ, причем более выраженное снижение этих показателей отмечалось среди пациентов ХОБЛ в условиях коморбидности с АГ. При этом статистически значимые межгрупповые различия среди пациентов, не имеющих группы инвалидности, получены по шкале общего здоровья.

Таблийа №2.

Сравнительный анализ показателей качества жизни в зависимости от группы инвалидности пациентов $\left(\mathrm{Me}\left[\mathrm{Q}_{25}-\mathrm{Q}_{75}\right]\right)$

\begin{tabular}{|c|c|c|c|c|c|c|}
\hline \multirow{2}{*}{ КЖ } & \multicolumn{3}{|c|}{$\begin{array}{c}1-я \text { группа ХОБЛ } \\
(\mathrm{n}=28)\end{array}$} & \multicolumn{3}{|c|}{$\begin{array}{c}2 \text {-я группа ХОБЛ }+ \text { АГ } \\
(\mathrm{n}=57)\end{array}$} \\
\hline & $\begin{array}{l}\text { 1. Нет груп- } \\
\text { пы }\end{array}$ & \begin{tabular}{|c|} 
2. III-я груп- \\
па \\
\end{tabular} & 3. II-я группа & $\begin{array}{l}\text { 1. Нет груп- } \\
\text { пы }\end{array}$ & $\begin{array}{l}\text { 2. III-я груп- } \\
\text { па } \\
\end{array}$ & 3. II-я группа \\
\hline \multirow[t]{2}{*}{ O3 } & $\begin{array}{c}57,5 \\
{[30,0-72,5]}\end{array}$ & $\begin{array}{c}27,0 \\
{[18,5-31,0]}\end{array}$ & $\begin{array}{c}42,5 \\
{[25,0-45,0]}\end{array}$ & $\begin{array}{c}35,0 \\
{[25,0-45,0]}\end{array}$ & $\begin{array}{c}35,0 \\
{[20,0-45,0]}\end{array}$ & $\begin{array}{c}30,0 \\
{[15,0-42,0]}\end{array}$ \\
\hline & \multicolumn{3}{|c|}{ pI $1,2=0,09$ pI $1,3=0,8$ pI $2,3=0,06$} & \multicolumn{3}{|c|}{ pII $1,2=0,6$ pII $1,3=0,08$ pII $2,3=0,06$} \\
\hline \multicolumn{7}{|c|}{$\mathbf{p 1} \mathbf{1 , 2}=\mathbf{0 , 0 3}$ p2 $1,2=0,8$ p3 $1,2=0,06$} \\
\hline \multirow[t]{2}{*}{ ФА } & $\begin{array}{c}72,5 \\
{[35,0-90,0]}\end{array}$ & $\begin{array}{c}40,0 \\
{[25,0-60,0]}\end{array}$ & $\begin{array}{c}22,5 \\
{[15,0-30,0]}\end{array}$ & $\begin{array}{c}55,0 \\
{[35,0-45,0]}\end{array}$ & $\begin{array}{c}35,0 \\
{[25,0-40,0]}\end{array}$ & $\begin{array}{c}25,0 \\
{[10,0-40,0]}\end{array}$ \\
\hline & \multicolumn{3}{|c|}{ pI $1,2=0,07$ pI $\mathbf{1 , 3}=\mathbf{0 , 0 3}$ pI $2,3=0,02$} & \multicolumn{3}{|c|}{ pI 1,2=0,04 pI 1,3=0,001 pI $2,3=0,02$} \\
\hline \multicolumn{7}{|c|}{ p1 $1,2=0,4$ p2 $1,2=0,6$ p3 $1,2=0,2$} \\
\hline \multirow[t]{2}{*}{ РФ } & $\begin{array}{c}50,0 \\
{[25,0-90,0]}\end{array}$ & $\begin{array}{c}25,0 \\
{[10,0-50,0]} \\
\end{array}$ & $\begin{array}{c}10,0 \\
{[5,0-30,0]} \\
\end{array}$ & $\begin{array}{c}50 \\
{[15,0-60,0]} \\
\end{array}$ & $\begin{array}{c}10,0 \\
{[5,0-25,0]}\end{array}$ & $\begin{array}{c}10,0 \\
{[5,0-15,0]}\end{array}$ \\
\hline & \multicolumn{3}{|c|}{ pI $1,2=0,07$ pI $\mathbf{1 , 3}=\mathbf{0 , 0 4}$ pI 2,3=0,06 } & \multicolumn{3}{|c|}{ pI $1,2=0,04$ pI $1,3=0,005$ pI $2,3=0,02$} \\
\hline \multicolumn{7}{|c|}{$\mathrm{p} 11,2=0,3 \mathrm{p} 21,2=0,1 \mathrm{p} 31,2=0,5$} \\
\hline \multirow[t]{2}{*}{ РЭ } & $\begin{array}{c}67,0 \\
{[34,0-95,0]}\end{array}$ & $\begin{array}{c}50,5 \\
{[17,0-83,5]} \\
\end{array}$ & $\begin{array}{c}10,0 \\
{[5,0-34,0]} \\
\end{array}$ & $\begin{array}{c}80,0 \\
{[34,0-95,0]} \\
\end{array}$ & $\begin{array}{c}15,0 \\
{[10,0-34,0]} \\
\end{array}$ & $\begin{array}{c}10,0 \\
{[5,0-34,0]}\end{array}$ \\
\hline & \multicolumn{3}{|c|}{ pI $1,2=0,07$ pI $\mathbf{1}, \mathbf{3}=\mathbf{0 , 0 2}$ pI $2,3=0,06$} & \multicolumn{3}{|c|}{ pI $1,2=\mathbf{0 , 0 2}$ pI $\mathbf{1 , 3}=\mathbf{0 , 0 0 1}$ pI $2,3=0,02$} \\
\hline
\end{tabular}


Окончание табл. 2.

\begin{tabular}{|c|c|c|c|c|c|c|}
\hline \multicolumn{7}{|c|}{ p1 $1,2=0,5$ p2 $1,2=0,4$ p3 $1,2=0,8$} \\
\hline \multirow[t]{2}{*}{$\mathrm{CA}$} & $\begin{array}{c}50,0 \\
{[38,0-55,0]}\end{array}$ & $\begin{array}{c}38,0 \\
{[30,0-50,5]}\end{array}$ & $\begin{array}{c}50,0 \\
{[38,0-55,0]}\end{array}$ & $\begin{array}{c}38,0 \\
{[30,0-50,0]}\end{array}$ & $\begin{array}{c}50,0 \\
{[38,0-63,0]}\end{array}$ & $\begin{array}{c}50,0 \\
{[38,0-55,0]}\end{array}$ \\
\hline & \multicolumn{3}{|c|}{ pI $1,2=0,7$ pI $1,3=0,3$ pI $2,3=0,6$} & \multicolumn{3}{|c|}{ pII $1,2=0,7$ pII $1,3=0,5$ pII $2,3=0,7$} \\
\hline \multicolumn{7}{|c|}{$\mathrm{p} 11,2=0,1 \mathrm{p} 21,2=0,9 \mathrm{p} 31,2=0,2$} \\
\hline \multirow[t]{2}{*}{ Б } & $\begin{array}{c}62,0 \\
{[41,0-74,0]}\end{array}$ & $\begin{array}{c}47,5 \\
{[36,5-77,0]}\end{array}$ & $\begin{array}{c}31,0 \\
{[30,0-32,0]}\end{array}$ & $\begin{array}{c}62,0 \\
{[41,0-74,0]}\end{array}$ & $\begin{array}{c}41,0 \\
{[22,0-52,0]}\end{array}$ & $\begin{array}{c}41,0 \\
{[31,0-54,0]}\end{array}$ \\
\hline & \multicolumn{3}{|c|}{ pI $1,2=0,5$ pI $\mathbf{1 , 3}=\mathbf{0 , 0 0 7}$ pI $\mathbf{2 , 3}=\mathbf{0 , 0 3}$} & \multicolumn{3}{|c|}{ pII $\mathbf{1 , 2}=\mathbf{0 , 0 3}$ pII $\mathbf{1 , 3}=\mathbf{0 , 0 2}$ pII $2,3=0,2$} \\
\hline \multicolumn{7}{|c|}{ p1 $1,2=0,5$ p2 $1,2=0,6$ p3 $1,2=0,4$} \\
\hline \multirow[t]{2}{*}{ ЖС } & $\begin{array}{c}57,5 \\
{[30,0-75,0]}\end{array}$ & $\begin{array}{c}27,5 \\
{[22,5-40,0]}\end{array}$ & $\begin{array}{c}35,0 \\
{[25,0-55,0]}\end{array}$ & $\begin{array}{c}50,0 \\
{[35,0-60,0]}\end{array}$ & $\begin{array}{c}40,0 \\
{[25,0-55,0]}\end{array}$ & $\begin{array}{c}30,0 \\
{[15,0-50,0]}\end{array}$ \\
\hline & \multicolumn{3}{|c|}{ pI $1,2=0,4$ pI $1,3=0,7$ pI $2,3=0,06$} & \multicolumn{3}{|c|}{ pII $1,2=0,7$ pII $\mathbf{1 , 3}=\mathbf{0 , 0 2}$ pII $2,3=0,006$} \\
\hline \multicolumn{7}{|c|}{ p1 1,2=0,6 p2 1,2=0,8 p3 1,2=0,1 } \\
\hline \multirow[t]{2}{*}{ ПЗ } & $\begin{array}{c}68,0 \\
{[28,0-76,0]}\end{array}$ & $\begin{array}{c}44,0 \\
{[36,0-60,0]}\end{array}$ & $\begin{array}{c}40,0 \\
{[35,0-52,0]}\end{array}$ & $\begin{array}{c}48,0 \\
{[36,0-68,0]}\end{array}$ & $\begin{array}{c}60,0 \\
{[28,0-72,0]}\end{array}$ & $\begin{array}{c}40,0 \\
{[24,0-52,0]}\end{array}$ \\
\hline & \multicolumn{3}{|c|}{ pI $1,2=0,3$ pI $1,3=0,8 \mathrm{pI} 2,3=0,06$} & \multicolumn{3}{|c|}{ pII $1,2=0,6$ pII $1,3=0,09$ pII $2,3=0,1$} \\
\hline & & & $=0,5 \mathrm{p} 21$, & $31,2=0,2$ & & \\
\hline
\end{tabular}

Примечание: ОЗ - общее здоровье, ФА - физическая активность, РФ - роль физических проблем в ограничении жизнедеятельности, РЭ-роль эмоциональных проблем в ограничении жизнедеятельности, СА - социальная активность, Б - боль, ЖС - жизнеспособность, ПЗ - психическое здоровье.

Так у пациентов 1-й группы О3 в среднем составляло 57,5 [30,0-72,5] баллов, у 2-й группы - 35,0 [25,0-45,0] баллов $(\mathrm{p}=0,03)$, соответственно, что, вероятно, обусловлено коморбидностью данных патологий и влиянием АГ на течение основного заболевания.

Проведен анализ влияния среднего прожиточного минимума на одного члена семьи на показатели КЖ (табл. 3).

Таблицุа №3.

Сравнительный анализ показателей качества жизни в зависимости от прожиточного минимума $\left(\mathrm{Me}\left[\mathrm{Q}_{25}-\mathrm{Q}_{75}\right]\right)$

\begin{tabular}{|c|c|c|c|c|c|c|}
\hline \multirow[t]{2}{*}{ КЖ } & \multicolumn{2}{|c|}{$\begin{array}{c}1 \text {-я группа ХОБЛ } \\
(\mathrm{n}=28)\end{array}$} & \multicolumn{2}{|c|}{$\begin{array}{c}2 \text { 2-я группа ХОБЛ + АГ } \\
(\mathrm{n}=57)\end{array}$} & \multicolumn{2}{|c|}{$\begin{array}{c}3 \text {-я группа АГ } \\
(\mathrm{n}=40)\end{array}$} \\
\hline & I. Средний & II. Низкий & I. Средний & II. Низкий & I. Средний & II. Низкий \\
\hline \multirow[t]{2}{*}{ O3 } & $\begin{array}{c}66,0 \\
{[40,0-77,0]} \\
\end{array}$ & $\begin{array}{c}37,5 \\
{[22,5-53,5]}\end{array}$ & $\begin{array}{c}35,0 \\
{[20,0-45,0]}\end{array}$ & $\begin{array}{c}35,0 \\
{[20,0-45,0]}\end{array}$ & $\begin{array}{c}60,0 \\
{[52,0-70,0]}\end{array}$ & $\begin{array}{c}52,0 \\
{[40,0-67,0]} \\
\end{array}$ \\
\hline & \multicolumn{2}{|c|}{$\mathrm{p}=\mathbf{0 , 0 3}$} & \multicolumn{2}{|c|}{$\mathrm{p}=0,6$} & \multicolumn{2}{|c|}{$\mathrm{p}=0,4$} \\
\hline \multicolumn{7}{|c|}{ pI $\mathbf{1}, \mathbf{2}=\mathbf{0 , 0 0 9}$ pI $1,3=0,8$ pI $\mathbf{2 , 3}=\mathbf{0 , 0 0 1}$ pII $1,2=0,6$ pII $\mathbf{1 , 3}=\mathbf{0 , 0 0 8}$ pII $\mathbf{2 , 3}=\mathbf{0 , 0 0 2}$} \\
\hline \multirow[t]{2}{*}{ ФА } & $\begin{array}{c}82,55 \\
{[50,0-95,0]} \\
\end{array}$ & $\begin{array}{c}35,0 \\
{[25,0-65,0]}\end{array}$ & $\begin{array}{c}35,0 \\
{[20,0-65,0]}\end{array}$ & $\begin{array}{c}35,0 \\
{[20,0-45,0]}\end{array}$ & $\begin{array}{c}90,0 \\
{[80,0-95,0]} \\
\end{array}$ & $\begin{array}{c}92,5 \\
{[85,0-95,0]} \\
\end{array}$ \\
\hline & \multicolumn{2}{|c|}{$\mathrm{p}=\mathbf{0 , 0 2}$} & \multicolumn{2}{|c|}{$\mathrm{p}=0,8$} & \multicolumn{2}{|c|}{$\mathrm{p}=0,7$} \\
\hline
\end{tabular}


Окончание табл. 3.

\begin{tabular}{|c|c|c|c|c|c|c|}
\hline \multicolumn{7}{|c|}{ pI $1,2=0,09$ pI $\mathbf{1}, \mathbf{3}=\mathbf{0 , 0 5}$ pI 2,3=0,001 pII $1,2=0,3$ pII $\mathbf{1 , 3}=\mathbf{0 , 0 1}$ pII $\mathbf{2 , 3}=\mathbf{0 , 0 0 2}$} \\
\hline \multirow[t]{2}{*}{ РФ } & $\begin{array}{c}50,0 \\
{[10,0-90,0]}\end{array}$ & $\begin{array}{c}25,0 \\
{[10,0-50,0]}\end{array}$ & $\begin{array}{c}15,0 \\
{[10,0-50,0]}\end{array}$ & $\begin{array}{c}10,0 \\
{[5,0-25,0]}\end{array}$ & $\begin{array}{c}75,0 \\
{[25,0-90,0]}\end{array}$ & $\begin{array}{c}50,0 \\
{[25,0-95,0]}\end{array}$ \\
\hline & \multicolumn{2}{|c|}{$\mathrm{p}=0,4$} & \multicolumn{2}{|c|}{$\mathrm{p}=0,4$} & \multicolumn{2}{|c|}{$\mathrm{p}=0,5$} \\
\hline \multicolumn{7}{|c|}{ pI $1,2=0,07$ pI 1,3=0,9 pI $\mathbf{2 , 3}=\mathbf{0 , 0 1}$ pII $1,2=0,4$ pII $\mathbf{1 , 3}=\mathbf{0 , 0 5}$ pII $\mathbf{2 , 3}=\mathbf{0 , 0 2}$} \\
\hline \multirow{2}{*}{ РЭ } & $\begin{array}{c}90,0 \\
{[34,0-95,0]}\end{array}$ & $\begin{array}{c}34,0 \\
{[10,0-67,0]}\end{array}$ & $\begin{array}{c}34,0 \\
{[20,0-90,0]}\end{array}$ & $\begin{array}{c}34,0 \\
{[10,0-67,0]}\end{array}$ & $\begin{array}{c}80,0 \\
{[34,0-95,0]}\end{array}$ & $\begin{array}{c}67,0 \\
{[34,0-95,0]}\end{array}$ \\
\hline & \multicolumn{2}{|c|}{$\mathrm{p}=0,09$} & \multicolumn{2}{|c|}{$\mathrm{p}=0,2$} & \multicolumn{2}{|c|}{$\mathrm{p}=0,5$} \\
\hline \multicolumn{7}{|c|}{ pI $1,2=0,3$ pI $1,3=0,5$ pI $2,3=0,6$ pII $1,2=0,6$ pII $\mathbf{1}, \mathbf{3}=\mathbf{0 , 0 3}$ pII $\mathbf{2 , 3}=\mathbf{0 , 0 2}$} \\
\hline \multirow{2}{*}{$\mathrm{CA}$} & $\begin{array}{c}50,0 \\
{[38,0-60,0]}\end{array}$ & $\begin{array}{c}50,0 \\
{[38,0-55,0]}\end{array}$ & $\begin{array}{c}50,0 \\
{[38,0-63,0]}\end{array}$ & $\begin{array}{c}40,0 \\
{[38,0-50,0]}\end{array}$ & $\begin{array}{c}50,0 \\
{[38,0-60,0]}\end{array}$ & $\begin{array}{c}46,0 \\
{[38,0-50,0]}\end{array}$ \\
\hline & \multicolumn{2}{|c|}{$\mathrm{p}=0,9$} & \multicolumn{2}{|c|}{$\mathrm{p}=0,2$} & \multicolumn{2}{|c|}{$\mathrm{p}=0,8$} \\
\hline \multicolumn{7}{|c|}{ pI $1,2=0,7$ pI $1,3=0,3$ pI $2,3=0,6$ pII $1,2=0,7$ pII $1,3=0,5$ pII $2,3=0,7$} \\
\hline \multirow[t]{2}{*}{ Б } & $\begin{array}{c}62,0 \\
{[36,0-92,0]}\end{array}$ & $\begin{array}{c}46,0 \\
{[31,0-74,0]}\end{array}$ & $\begin{array}{c}41,0 \\
{[40,0-62,0]}\end{array}$ & $\begin{array}{c}41,0 \\
{[31,0-62,0]}\end{array}$ & $\begin{array}{c}62,0 \\
{[41,0-90,0]}\end{array}$ & $\begin{array}{c}74,0 \\
{[52,0-95,0]}\end{array}$ \\
\hline & \multicolumn{2}{|c|}{$\mathrm{p}=0,4$} & \multicolumn{2}{|c|}{$\mathrm{p}=0,9$} & \multicolumn{2}{|c|}{$\mathrm{p}=0,9$} \\
\hline \multicolumn{7}{|c|}{ pI $1,2=0,5$ pI $1,3=0,4$ pI $2,3=0,06$ pII $1,2=0,5$ pII $1,3=0,4$ pII $2,3=0,2$} \\
\hline \multirow[t]{2}{*}{ ЖС } & $\begin{array}{c}72,5 \\
{[22,5-77,5]}\end{array}$ & $\begin{array}{c}37,5 \\
{[25,0-57,5]}\end{array}$ & $\begin{array}{c}45,0 \\
{[35,0-60,0]}\end{array}$ & $\begin{array}{c}35,0 \\
{[25,0-50,0]} \\
\end{array}$ & $\begin{array}{c}65,0 \\
{[50,0-75,0]}\end{array}$ & $\begin{array}{c}55,0 \\
{[35,0-80,0]}\end{array}$ \\
\hline & \multicolumn{2}{|c|}{$\mathrm{p}=0,19$} & \multicolumn{2}{|c|}{$\mathrm{p}=0,1$} & \multicolumn{2}{|c|}{$\mathrm{p}=0,4$} \\
\hline \multicolumn{7}{|c|}{ pI $1,2=0,4$ pI $1,3=0,7$ pI $\mathbf{2 , 3}=\mathbf{0 , 0 0 4}$ pII $1,2=0,7$ pII $\mathbf{1}, \mathbf{3}=\mathbf{0 , 0 1}$ pII $\mathbf{2 , 3}=\mathbf{0 , 0 0 6}$} \\
\hline \multirow[t]{2}{*}{ ПЗ } & $\begin{array}{c}75,5 \\
{[34,0-78,0]}\end{array}$ & $\begin{array}{c}44,0 \\
{[36,0-68,0]}\end{array}$ & $\begin{array}{c}52,0 \\
{[36,0-64,0]}\end{array}$ & $\begin{array}{c}44,0 \\
{[28,0-64,0]}\end{array}$ & $\begin{array}{c}66,0 \\
{[48,0-80,0]}\end{array}$ & $\begin{array}{c}60,0 \\
{[52,0-84,0]}\end{array}$ \\
\hline & \multicolumn{2}{|c|}{$\mathrm{p}=0,2$} & \multicolumn{2}{|c|}{$\mathrm{p}=0,4$} & \multicolumn{2}{|c|}{$\mathrm{p}=0,8$} \\
\hline & & I $1,3=0$, & $\epsilon=0,00$ & $2=0,6$ pII 1 , &, 09 pII 2, & \\
\hline
\end{tabular}

Примечание: ОЗ - общее здоровье, ФА - физическая активность, РФ - роль физических проблем в ограничении жизнедеятельности, РЭ-роль эмоциональных проблем в ограничении жизнедеятельности, СА - социальная активность, Б - боль, ЖС - жизнеспособность, ПЗ - психическое здоровье.

Выявлены внутригрупповые статистически значимые различия $(\mathrm{p}<0,05)$ по шкале общего здоровья и физического функционирования среди пациентов с изолированной ХОБЛ, показатели которых были ниже у лиц с низким прожиточным минимумом, по остальным шкалам отмечалась тенденция к снижению всех показателей. Статистически значимых различий среди пациентов $2^{\text {-й }}$ группы получено не было, что возможно обусловлено коморбидностью данных патологий и влиянием АГ на течение ХОБЛ. Сопоставление межгрупповых результатов по отдельным шкалам опросника SF-36 показало, что в большей степени на уровень КЖ влияет ХОБЛ, при этом как физический, так и психоэмоциональный статус респондентов. Однако следует отметить, что у лиц с низким прожиточным 
минимумом имеется тенденция к более низким показателям КЖ по всем шкалам опросника SF-36. Подводя итог установлено, что социально-экономический статус оказывает существенное влияние на КЖ респондентов, при чем как в целом, так и на отдельно составляющие его части.

\section{Выводы}

В ходе проведенного исследования выявлено снижение КЖ по всем параметрам у пациентов с ХОБЛ и в большей степени у лиц при коморбидности ХОБЛ и АГ, что вероятнее всего связано с прогрессированием и большей выраженностью клинической симптоматики. Наиболее значимой сферой отношений на всех стадиях является социально-экономический статус, значимость которого увеличивается по мере нарастания тяжести болезни. Более низкие значения по шкалам, характеризующим как физический, так и психосоциальный статус респондентов, регистрировались у неработающих лиц. КЖ прямо пропорционально снижалось $(\mathrm{p}<0,05)$, при наличии группы инвалидности, при чем более выраженно у пациентов при коморбидности ХОБЛ и АГ. Рассматривая влияние среднего прожиточного минимума, из расчета на одного члена семьи, на уровень КЖ, статистически значимые внутригрупповые отличия были получены среди пациентов с ХОБЛ без АГ по шкалам общего здоровья и физического функционирования $(\mathrm{p}<0,05)$.

\section{Список литературы}

1. Алексеев И.А., Харьков Е.И., Иванов А.Г. и др. Особенности показателей качества жизни до и после проведения школы по артериальной гипертонии у лиц пожилого и старческого возраста // В мире научных открытий. 2018. T. 10, № 4. C. 204-222.

2. Асланян Я.А., Перцева М.В., Перцев А.В. Оценка качества жизни пациентов с хронической обструктивной болезнью легких на фоне применения комплексной лечебно-профилактической программы // Молодежный инновационный вестник. 2018. T. 7. № S1. С. 54-55.

3. Ахмедова Э.Б., Марданов Б.У., Бадейникова К.К., Мамедов М.Н. Исследование качества жизни и параметров клинико-биохимического спектра у больных артериальной гипертензией и коморбидными состояниями // Рациональная фармакотерапия в кардиологии. 2017. Т. 13, № 1. С. 31-35.

4. Глобальная стратегия диагностики, лечения и профилактики хронической обструктивной болезни легких (пересмотр 2014 г.) / Пер. с англ. под ред. А.С. Белевского. М.: Российское респираторное общество, 2014. 92 с. 
5. Давыдов Е.Л. Вопросы тревожно-депрессивных состояний у больных артериальной гипертонией старших возрастных групп // Клиническая геронтология. 2016. Т. 22, № 9-10. С. 22-23.

6. Давыдов Е.Л., Ульянова И.О. Медико-социальные аспекты больных артериальной гипертонией в пожилом возрасте // Клиническая геронтология. 2016. T. 22, № 9-10. C. 24-25.

7. Ермакова М.К., Капустина Н.Р., Ермаков Г.И. и др. Качество жизни как показатель степени адаптации к болезни на примере бронхиальной астмы у детей и хронической обструктивной болезни легких у взрослых // Общественное здоровье и здравоохранение. 2013. № 1. С. 65-71.

8. Игнатова Г.Л., Антонов В.Н., Родионова О.В. и др. Качество жизни пациентов с хронической обструктивной болезнью легких при двойной бронходилатационной терапии // Практическая пульмонология. 2016. № 4. C. 9-14.

9. Козлов Е.В. Структурно-функциональные изменения сердечно-сосудистой системы у пациентов, страдающих артериальной гипертонией в сочетании с хронической обструктивной болезнью легких // Сибирское медицинское обозрение. 2016. № 3 (99). С. 56-66.

10. Козлов Е.В. Тревожно-депрессивные расстройства при коморбидности хронической обструктивной болезни легких и артериальной гипертонии // Сибирское медицинское обозрение. 2014. № 1 (85). С. 19-25.

11. Козлов Е.В., Деревянных Е.В., Балашова Н.А. и др. Хроническая обструктивная болезнь легких как социально-экономическое бремя взрослого населения (научный обзор) // В мире научных открытий. 2018. Т. 10, № 3. C. $180-199$.

12. Козлов Е.В., Петрова М.М., Харьков Е.И. Качество жизни у больных хронической обструктивной болезнью легких в сочетании с артериальной гипертонией // Забайкальский медицинский вестник. 2014. №4. С. 6-13.

13. Ремизов Е.В., Давыдов Е.Л., Корепина Т.В. Проблемы качества жизни пациентов старших возрастных групп с артериальной гипертонией // Военно-медицинский журнал. 2012. № 5. С. 64.

14. Степашкин К.Н., Петрова М.М., Демко И.В. Влияние коморбидной кардиоваскулярной патологии на качество жизни больных хронической обструктивной болезнью легких // Сибирское медицинское обозрение. 2013. № 4 (82). C. 66-69.

15. Филимонова Л.А., Борисенко Н.А., Алексеев И.А. и др. Мониторинг качества жизни у пациентов артериальной гипертонии старших возрастных групп // Современные проблемы науки и образования. 2017. № 1. С. 2. 
16. Чазова И.Е., Чучалин А.Г., Зыков К.А., Ратова Л.Г. Диагностика и лечение пациентов с артериальной гипертонией и хронической обструктивной болезнью легких (Рекомендации Российского медицинского общества по артериальной гипертонии и Российского респираторного общества // Системные гипертензии. 2013. №1. С. 5-34.

17. Яскевич Р.А. Особенности влияния нейросенсорной тугоухости на качество жизни у пожилых мигрантов Крайнего Севера // Клиническая геронтология. 2016. Т. 22, № 9-10. С. 73-74.

18. Яскевич Р.А. Особенности показателей качества жизни в различные периоды реадаптации к новым климатическим условиям у пожилых мигрантов Крайнего Севера с артериальной гипертонией // Клиническая геронтология. 2017. Т. 23, № 9-10. С. 81-83.

19. Яскевич Р.А., Игнатова И.А., Шилов С.Н. и др. Влияние тревожно-депрессивных расстройств на качество жизни слабослышащих мигрантов Крайнего Севера в период реадаптации к новым климатическим условиям // Современные проблемы науки и образования. 2014. № 4. С. 279.

20. Arabyat R.M., Raisch D.W. Relationships Between Social/Emotional Support and Quality of Life, Depression and Disability in Patients With Chronic Obstructive Pulmonary Disease: An Analysis Based on Propensity Score Matching // Ann. Behav. Med. 2019. Vol.19. pii: kaz002.

21. Boueri F.M. Quality of life measured with a generic instrument (Short Form-36) improves following pulmonary rehabilitation in patients with COPD // Chest. 2001. Vol. 119. P. 77-84.

22. Celli B., Blasi F., Gaga M. et al. Perception of symptoms and quality of life comparison of patients and physicians views in the COPD MIRROR study. // Int. J. Chron. Obstruct. Pulmon. Dis. 2017. Vol.12. P. 2189-2196.

23. Jones G.L. Quality of life changes over time in patients with chronic obstructive pulmonary disease // Curr. Opin. Pulm. Med. 2016. Vol.22, N.2. P. 125-129.

24. Kontodimopoulos N., Stamatopoulou E., Brinia A. et al. Are condition-specific utilities more valid than generic preference-based ones in asthma? Evidence from a study comparing EQ-5D-3L and SF-6D with AQL-5D // Expert Rev Pharmacoecon Outcomes Res. 2018. Vol.18, N.6. P. 667-675.

25. Milanowska J., Mackiewicz B., Węgorowski P. et al. The quality of life of farmers with chronic obstructive pulmonary disease (COPD) // Annals of Agricultural and Environmental Medicine. 2017. Vol.24, N.2. P. 283-287.

26. Mroczek B., Sitko Z., Augustyniuk K. et al. Socioeconomic Indicators Shaping Quality of Life and Illness Acceptance in Patients with Chronic Obstructive Pulmonary Disease // Adv. Exp. Med. Biol. 2015. Vol.861. P. 19-30. 


\section{References}

1. Alekseev I.A., Har'kov E.I., Ivanov A.G. i dr. Osobennosti pokazatelej kachestva zhizni do i posle provedeniya shkoly po arterial'noj gipertonii u lic pozhilogo i starcheskogo vozrasta [Features of quality of life indicators before and after school for arterial hypertension in elderly and senile people]. V mire nauchnyh otkrytij [In the world of scientific discoveries]. 2018. V. 10. № 4. P. 204-222.

2. Aslanyan YA.A., Perceva M.V., Percev A.V. Ocenka kachestva zhizni pacientov s hronicheskoj obstruktivnoj bolezn'yu legkih na fone primeneniya kompleksnoj lechebno-profilakticheskoj programmy [Assessment of the quality of life of patients with chronic obstructive pulmonary disease on the background of the application of a comprehensive treatment and prevention program]. Molodezhnyj innovacionnyj vestnik [Molodezhny Innovative Bulletin]. 2018. V. 7. № S1. P. 54-55.

3. Ahmedova EH.B., Mardanov B.U., Badejnikova K.K., Mamedov M.N. Issledovanie kachestva zhizni i parametrov kliniko-biohimicheskogo spektra u bol'nyh arterial'noj gipertenziej i komorbidnymi sostoyaniyami [Study of the quality of life and parameters of the clinical and biochemical spectrum in patients with arterial hypertension and comorbid conditions]. Racional'naya farmakoterapiya v kardiologii [Rational pharmacotherapy in cardiology]. 2017. V. 13. № 1. P. 31-35.

4. Global'naya strategiya diagnostiki, lecheniya i profilaktiki hronicheskoj obstruktivnoj bolezni legkih (peresmotr 2014 g.) [The global strategy for the diagnosis, treatment and prevention of chronic obstructive pulmonary disease (revision 2014)]. Per. s angl. pod red. A.S. Belevskogo. M.: Russian Respiratory Society. 2014. 92 p.

5. Davydov E.L. Voprosy trevozhno-depressivnyh sostoyanij u bol'nyh arterial'noj gipertoniej starshih vozrastnyh grupp [Issues of anxiety-depressive states in patients with arterial hypertension of older age groups]. Klinicheskaya gerontologiya [Clinical gerontology]. 2016. V. 22. № 9-10. P. 22-23.

6. Davydov E.L., Ul'yanova I.O. Mediko-social'nye aspekty bol'nyh arterial'noj gipertoniej v pozhilom vozraste [Medical and social aspects of patients with arterial hypertension in old age]. Klinicheskaya gerontologiya [Clinical gerontology]. 2016. V. 22. № 9-10. P. 24-25.

7. Ermakova M.K., Kapustina N.R., Ermakov G.I. i dr. Kachestvo zhizni kak pokazatel' stepeni adaptacii k bolezni na primere bronhial'noj astmy u detej i hronicheskoj obstruktivnoj bolezni legkih u vzroslyh [Quality of life as an indicator of the degree of adaptation to the disease on the example of bronchial asthma in children and chronic obstructive pulmonary disease in adults]. Obshchest- 
vennoe zdorov'e i zdravoohranenie [Public Health and Health Care]. 2013. № 1. P. 65-71.

8. Ignatova G.L., Antonov V.N., Rodionova O.V. i dr. Kachestvo zhizni pacientov s hronicheskoj obstruktivnoj bolezn'yu legkih pri dvojnoj bronhodilatacionnoj terapii [The quality of life of patients with chronic obstructive pulmonary disease with dual bronchodilation therapy]. Prakticheskaya pul'monologiya [Practical pulmonology]. 2016. № 4. P. 9-14.

9. Kozlov E.V. Strukturno-funkcional'nye izmeneniya serdechno-sosudistoj sistemy u pacientov, stradayushchih arterial'noj gipertoniej v sochetanii s hronicheskoj obstruktivnoj bolezn'yu legkih [Structural and functional changes in the cardiovascular system in patients suffering from arterial hypertension in combination with chronic obstructive pulmonary disease]. Sibirskoe medicinskoe obozrenie [Siberian Medical Review]. 2016. № 3 (99). P. 56-66.

10. Kozlov E.V. Trevozhno-depressivnye rasstrojstva pri komorbidnosti hronicheskoj obstruktivnoj bolezni legkih i arterial'noj gipertonii [Anxiety-depressive disorders in comorbidity of chronic obstructive pulmonary disease and arterial hypertension]. Sibirskoe medicinskoe obozrenie [Siberian Medical Review]. 2014. № 1 (85). S. 19-25.

11. Kozlov E.V., Derevyannyh E.V., Balashova N.A. et al. Hronicheskaya obstruktivnaya bolezn' legkih kak social'no-ehkonomicheskoe bremya vzroslogo naseleniya (nauchnyj obzor) [Chronic obstructive pulmonary disease as a socio-economic burden of the adult population (scientific review)]. $V$ mire nauchnyh otkrytij [In the world of scientific discoveries]. 2018. V. 10, № 3. P. $180-199$.

12. Kozlov E.V., Petrova M.M., Har'kov E.I. Kachestvo zhizni u bol'nyh hronicheskoj obstruktivnoj bolezn'yu legkih v sochetanii s arterial'noj gipertoniej [Quality of life in patients with chronic obstructive pulmonary disease in combination with arterial hypertension]. Zabajkal'skij medicinskij vestnik [Transbaikalian Medical Journal]. 2014. № 4. P. 6-13.

13. Remizov E.V., Davydov E.L., Korepina T.V. Problemy kachestva zhizni pacientov starshih vozrastnyh grupp s arterial'noj gipertoniej [Problems of quality of life of patients in older age groups with arterial hypertension]. Voenno-medicinskij zhurnal [Military Medical Journal]. 2012. № 5. P. 64.

14. Stepashkin K.N., Petrova M.M., Demko I.V. Vliyanie komorbidnoj kardiovaskulyarnoj patologii na kachestvo zhizni bol'nyh hronicheskoj obstruktivnoj bolezn'yu legkih [Influence of comorbid cardiovascular pathology on the quality of life of patients with chronic obstructive pulmonary disease]. Sibirskoe medicinskoe obozrenie [Siberian Medical Review]. 2013. № 4 (82). P. 66-69. 
15. Filimonova L.A., Borisenko N.A., Alekseev I.A. i dr. Monitoring kachestva zhizni u pacientov arterial'noj gipertonii starshih vozrastnyh grupp [Monitoring the quality of life in patients with arterial hypertension in older age group]. Sovremennye problemy nauki i obrazovaniya [Modern problems of science and education]. 2017. № 1. P. 2.

16. Chazova I.E., Chuchalin A.G., Zykov K.A., Ratova L.G. Diagnostika i lechenie pacientov s arterial'noj gipertoniej i hronicheskoj obstruktivnoj bolezn'yu legkih (Rekomendacii Rossijskogo medicinskogo obshchestva po arterial'noj gipertonii i Rossijskogo respiratornogo obshchestva [Diagnostics and treatment of patients with arterial hypertension and chronic obstructive pulmonary disease (Recommendations of the Russian Medical Society on arterial hypertension and the Russian Respiratory Society]. Sistemnye gipertenzii [Systemic hypertension]. 2013. №1. P. 5-34.

17. Yaskevich R.A. Osobennosti vliyaniya nejrosensornoj tugouhosti na kachestvo zhizni u pozhilyh migrantov Krajnego Severa [Features of the impact of neurosensory hearing loss on the quality of life in elderly migrants of the Far North]. Klinicheskaya gerontologiya [Clinical gerontology]. 2016. V. 22. № 9-10. P. 73-74.

18. Yaskevich R.A. Osobennosti pokazatelej kachestva zhizni v razlichnye periody readaptacii k novym klimaticheskim usloviyam u pozhilyh migrantov Krajnego Severa s arterial'noj gipertoniej [Features of indicators of quality of life in different periods of readaptation to new climatic conditions in elderly migrants of the Far North with arterial hypertension]. Klinicheskaya gerontologiya [Clinical gerontology]. 2017. V. 23. № 9-10. P. 81-83.

19. Yaskevich R.A., Ignatova I.A., Shilov S.N. i dr. Vliyanie trevozhno-depressivnyh rasstrojstv na kachestvo zhizni slaboslyshashchih migrantov Krajnego Severa $\mathrm{v}$ period readaptacii k novym klimaticheskim usloviyam [The influence of anxiety-depressive disorders on the quality of life of hearing impaired migrants of the Far North in the period of adaptation to new climatic conditions]. Sovremennye problemy nauki i obrazovaniya [Modern problems of science and education]. 2014. № 4. P. 279.

20. Arabyat R.M., Raisch D.W. Relationships Between Social/Emotional Support and Quality of Life, Depression and Disability in Patients With Chronic Obstructive Pulmonary Disease: An Analysis Based on Propensity Score Matching. Ann. Behav. Med. 2019. Vol.19. pii: kaz002.

21. Boueri F.M. Quality of life measured with a generic instrument (Short Form36) improves following pulmonary rehabilitation in patients with COPD. Chest. 2001. Vol. 119. P. 77-84. 
22. Celli B., Blasi F., Gaga M. et al. Perception of symptoms and quality of life comparison of patients and physicians views in the COPD MIRROR study. Int. J. Chron. Obstruct. Pulmon. Dis. 2017. Vol.12. P. 2189-2196.

23. Jones G.L. Quality of life changes over time in patients with chronic obstructive pulmonary disease. Curr. Opin. Pulm. Med. 2016. Vol.22. N.2. P. 125-129.

24. Kontodimopoulos N., Stamatopoulou E., Brinia A. et al. Are condition-specific utilities more valid than generic preference-based ones in asthma? Evidence from a study comparing EQ-5D-3L and SF-6D with AQL-5D. Expert Rev Pharmacoecon Outcomes Res. 2018. Vol.18. N.6. P. 667-675.

25. Milanowska J., Mackiewicz B., Węgorowski P. et al. The quality of life of farmers with chronic obstructive pulmonary disease (COPD). Annals of Agricultural and Environmental Medicine. 2017. Vol.24. N.2. P. 283-287.

26. Mroczek B., Sitko Z., Augustyniuk K. et al. Socioeconomic Indicators Shaping Quality of Life and Illness Acceptance in Patients with Chronic Obstructive Pulmonary Disease. Adv. Exp. Med. Biol. 2015. Vol.861. P. 19-30.

\section{ДАННЫЕ ОБ АВТОРАХ}

Козлов Евгений Вячеславович, доцент кафедры пропедевтики внутренних болезней и терапии, заведующий пульмонологическим отделением, кандидат медицинских наук

ГБОУ ВПО «КрасГМУ им. проф. Ф.В. Войно-Ясенеикого» МЗ РФ; КГБУЗ «Красноярская межрайонная клиническая больнииа скорой медииинской помощи имени Н.С. Карповича» ул. Партизана Железняка, 1а, Красноярск, 660022, Российская Федерачия; ул. Курчатова, 17, г. Красноярск, 660062, Российская Федерачия kev-pulmonolog@mail.ru

Деревянных Евгений Валерьевич, доцент кафедры пропедевтики внутренних болезней и терапии, кандидат медицинских наук ГБОУ ВПО «КрасГМУ им. проф. Ф.В. Войно-Ясенечкого» МЗ РФ ул. Партизана Железняка, 1а, Красноярск, 660022, Российская Федерачия rusene@mail.ru

Балашова Наталья Арленовна, доцент кафедры пропедевтики внутренних болезней и терапии, кандидат медицинских наук, доцент ГБОУ ВПО «КрасГМУ им. проф. Ф.В. Войно-Ясенечкого» МЗ РФ 
ул. Партизана Железняка, 1а, Красноярск, 660022, Российская Федерачия

balashova-61@mail.ru

Яскевич Роман Анатольевич, доцент кафедры пропедевтики внутренних болезней и терапии, ведущий научный сотрудник группы патологии сердечно-сосудистой системы, кандидат медицинских наук, доцент ГБОУ ВПО «КрасГМУ им. проф. Ф.В. Войно-Ясенеикого» МЗ РФ; Федеральное государственное бюджетное научное учреждение «Научно-исследовательский институт медииинских проблем Севера» ул. Партизана Железняка, 1а, Красноярск, 660022, Российская Федераџия; ул. Партизана Железняка, 32, Красноярск, 660022, Российская Федерация cardio@impn.ru

Москаленко Ольга Леонидовна, старший научный сотрудник, кандидат биологических наук Федеральное государственное бюджетное научное учреждение «Научно-исследовательский институт медицинских проблем Севера» ул. Партизана Железняка, 32, Красноярск, 660022, Российская Федерачия gre-ll@mail.ru

\section{DATA ABOUT THE AUTHORS}

Kozlov Evgeny Vyacheslavovich, associate professor at department of propedeutics of internal diseases and therapy, head of pulmonology department, candidate of medical science

Krasnoyarsk State Medical University named after Professor V.F. VoinoYasenetzkiy; Krasnoyarsk Interdistrict Clinical Hospital of Emergency Medical Care. N.S. Karpovich

1a, P. Zheleznyaka St., Krasnoyarsk, 660022, Russian Federation; 17, Kurchatov St., Krasnoyarsk, 660062, Russian Federation kev-pulmonolog@mail.ru

Derevyannich Evgeny Valeryevich, associate professor at department of propedeutics of internal diseases and therapy, candidate of medical science Krasnoyarsk State Medical University named after Professor V.F. Voino-Yasenetzkiy 
1a, P. Zheleznyaka St., Krasnoyarsk, 660022, Russian Federation rusene@mail.ru

Balashova Natalia Arlenovna, associate professor at department of propedeutics of internal diseases and therapy, candidate of medical science, docent

Krasnoyarsk State Medical University named after Professor V.F. Voino-Yasenetzkiy

1a, P. Zheleznyaka St., Krasnoyarsk, 660022, Russian Federation balashova-61@mail.ru

Yaskevich Roman Anatolyevich, associate professor at department of propedeutics of internal diseases and therapy, leading researcher of the group pathology of the cardiovascular system, candidate of medical science, docent

Krasnoyarsk State Medical University named after Professor V.F. Voino-Yasenetzkiy; Federal State Budgetary Scientific Institution «Scientific Research Institute of medical problems of the North 1a, P. Zheleznyaka St., Krasnoyarsk, 660022, Russian Federation; 3g, P. Zheleznyaka St., Krasnoyarsk, 660022, Russian Federation cardio@impn.ru

Moskalenko Olga Leonidovna, Senior Researcher, Candidate of Biological Sciences

Federal State Budgetary Scientific Institution «Scientific Research Institute of medical problems of the North»

3g, P. Zheleznyaka St., Krasnoyarsk, 660022, Russian Federation gre-ll@mail.ru 